\title{
A comparative analysis of radiographer versus radiologist in the diagnosis of rotator cuff tears of the shoulder using ultrasound
}

This article was published in the following Dove Press journal:

Orthopedic Research and Reviews

28 October 2015

Number of times this article has been viewed

\section{Zuned A Hakim \\ Syed Ali \\ R Stephen Bale \\ Peter J Hughes}

Trauma and Orthopaedic Department, Royal Preston Hospital, Preston, UK

Correspondence: Zuned A Hakim

Flat 4, Glenhaven House,

10-12 Clyde Road, Didsbury,

Manchester M20 2WH, UK

Email zuned.hakim@gmail.com
Introduction: Rotator cuff pathology is a commonly encountered shoulder complaint, which is routinely investigated with ultrasound scan. Sensitivities and specificities for detecting tears have been reported to be between $79 \%$ and $100 \%$. Our aim was to compare the scans performed by a radiographer versus a radiologist with that of surgery.

Patients and methods: This study is a retrospective review of 184 cases over a 12-month period who underwent arthroscopy following an ultrasound scan for cuff disease. Single clinician collected data for cuff pathology based on the scan report and compared to intraoperative findings. StatsDirect was used for statistical analysis to determine sensitivities, specificities, and accuracy.

Results: The radiologist had better sensitivity for partial-thickness tears by 33\% (95\% CI $4.4 \%-59.9 \%$, $P=0.018$ ). The radiographer had better specificity by $24 \%$ (CI $11.9 \%-38.8 \%, P=0.0001$ ). The radiographer had better accuracy at $55 \%$ versus radiologist at $23 \%$ and was better at quantifying the size of the tear. Intraoperative change in the plan occurred in $15 \%$ of cases in each group.

Discussion: Our findings for specificity, sensitivity, and accuracy for full-thickness tears are comparable to the existing literature, but lower than most of the quoted papers for partial-thickness tears. Change in intraoperative plan as a result of differing findings at surgery is equal in each group. We found the radiographer to be better at sizing the full-thickness tears.

Keywords: rotator cuff, ultrasound, accuracy difference

\section{Introduction}

Rotator cuff pathology in the shoulder is a frequent problem encountered in the shoulder speciality clinic. Pathology ranges from tendinopathy to full-thickness retracted tears. It is reasonable to quantify the clinical findings with the investigative imaging to aid in the formulation of a tailored management plan.

Ultrasound scan (USS) evaluation of the shoulder has been a technique used as an adjunct to clinical assessment since Seltzer et al first described its use to detect effusions in $1979 .{ }^{1}$ The advancement of technology has led to better quality transducers and improved image resolution, resulting in the routine use of ultrasound in the evaluation of the shoulder and the rotator cuff. It is relatively inexpensive, portable, and allows the dynamic functional assessment of the rotator cuff.

It is now becoming a common practice for trained musculoskeletal radiographers to undertake USS procedures in addition to medically trained musculoskeletal radiologists. A radiographer is a nonmedically trained practitioner, whereas a radiologist is a fully qualified doctor specializing in radiology.

The aim of this study was to determine the sensitivity, specificity, and accuracy of shoulder ultrasound in diagnosing rotator cuff tears and compare the findings 
of a trained consultant radiologist with that of a trained radiographer, in addition to determine the intraoperative change in plan that occurs due to incorrect information.

\section{Materials and methods}

We retrospectively collected data for all eligible patients. Using our Bluespier orthopedic patient management software, we identified 561 cases of shoulder arthroscopies operated by the two senior authors (SB, PH) over a 12-month period. The inclusion criteria were primary cases, cuff pathology on USS followed by surgery, and surgery within 4 months of USS; exclusion criteria were cases with other known pathology, surgery time $>4$ months following USS, and revision cases. Institutional Review Board approval was not deemed necessary for the following retrospective review. All principles outlined in the Declaration of Helsinki were followed.

Clinical notes and radiology reports were used for data collection, which was performed by a single clinician. We identified 561 cases, of which 377 were excluded to leave 184 for analysis. The reasons for exclusions were as follows: 139 - no USS, 135 - duplicate records, 40 - frozen shoulders, 21 - previous surgery, 22 - instability, 9 - nonshoulder pathology, 8 - USS to surgery time $>4$ months, 1 - incomplete operation record, 1 - open procedure, and 1 - osteoarthritis.

The mean age was 56 years (range 25-99 years). There were 89 women and 95 men. One hundred and twenty patients were scanned by the radiographer and 64 by the consultant radiologist. The mean time from ultrasound until surgery was 6.2 weeks (range 1-12 weeks).

The USS was performed by either a trained radiographer or a consultant radiologist with a musculoskeletal interest in the outpatient radiology department. Each performed the scan using the same method according to the departmental standardized technique for shoulders. The USS was interpreted by the person performing the scan who provided a written report of his/her findings. USS reports were analyzed to identify the person performing the scan and the condition of the rotator cuff, which was classified into tendinopathic, calcific, partial tear, and full-thickness tear. The size of the tear was grouped into $<1 \mathrm{~cm}, 1-3 \mathrm{~cm}, 3-5 \mathrm{~cm}$, or $>5 \mathrm{~cm}$. This measure was the retraction of the cuff from its insertion in the tuberosity. The surgeon was not blinded to the ultrasound report as it was used for the formulation of the management plan.

Two senior authors (SB, $\mathrm{PH}$ ) performed the arthroscopy or they were present at the time of surgery and made the decision regarding the condition of the rotator cuff. In cases where a fullthickness tear was present, its size from its insertion point was estimated from the bursal side using a hand probe of known length. Documentation was made using a standardized computer-generated template that allowed thorough documentation and classification of any tears according to their size and thickness. Postoperative management was in line with departmental protocol.

The data were analyzed using StatsDirect (StatsDirect Ltd, Chesire, UK) with confidence intervals set at 95\%. Sensitivities and specificities were calculated to determine the diagnostic capability of the radiographer and radiologist in relation to partial-thickness or full-thickness tears as well as to arrive at an overall figure. Accuracy was calculated with regard to the size of the tear.

\section{Results}

\section{Sensitivity and specificity}

Overall, 17 patients were reported as normal, of which two had a full-thickness tear and three had a partial tear and 12 were normal at surgery. Five patients were reported as calcification, of which two had a partial tear and three were normal at surgery. Seventy-nine patients were reported to have a full-thickness tear, of which 63 were full-thickness tears, seven were partial tears, seven were normal, and two were degenerative at surgery. Thirty-four patients were reported to have partial tears, of which four had a full-thickness tear, 12 had a partial tear, 17 were normal, and one was degenerative at surgery. Forty-nine patients were reported to be tendinopathic, of which one had a full-thickness tear, 20 had a partial tear, and 28 were normal.

A statistical analysis was performed by comparing the findings of the radiologist with the radiographer to determine the proportional difference. All results were insignificant other than sensitivity for partial-thickness tears, where the radiologist was better by $33 \%(\mathrm{CI} 4.4 \%-59.9 \%, P=0.018)$. The radiographer had better specificity by $24 \%$ (CI $11.9 \%-38.8 \%$, $P=0.0001$; Tables 1 and 2, and Figure 1A-D).

\section{Accuracy}

The accuracy for determining the correct size of a full-thickness tear was calculated. Overall, there were 70 full-thickness tears at surgery, of which 30 were correctly sized. The radiographer correctly sized 24 of the 44 full-thickness tears and the radiologist correctly sized six of the 26 full-thickness tears (Table 3 ).

\section{Difference in diagnostic rate}

A proportional analysis showed the radiographer to be better at quantifying the size by a difference of $31 \%$ (CI $8 \%-51 \%$; Figure 2). 
Table I Sensitivity and specificity of full-thickness tears

\begin{tabular}{lll}
\hline Full-thickness tears & Sensitivity & Specificity \\
\hline Overall results & $89 \%(\mathrm{Cl} 79 \%-96 \%)$ & $82 \%(\mathrm{Cl} 74 \%-79 \%)$ \\
Radiographer & $95 \%(\mathrm{Cl} 85 \%-99 \%)$ & $86 \%(\mathrm{Cl} \mathrm{76 \% -93 \% )}$ \\
Radiologist & $81 \%(\mathrm{Cl} 61 \%-93 \%)$ & $87 \%(\mathrm{Cl} \mathrm{72 \% -96 \% )}$ \\
\hline
\end{tabular}

Abbreviation: $\mathrm{Cl}$, confidence interval.

\section{Change in intraoperative plan}

Intraoperative change in the plan occurred in 18 of the 120 cases scanned by the radiographer. Fourteen were listed for a cuff repair, which was not required, and four were listed as noncuff repairs, which were required. Ten of the 64 cases scanned by the radiologist required an intraoperative change in the plan. Seven were listed for a cuff repair, which was not required, and three were listed as noncuff repairs, which were required. This gives an intraoperative change in the plan of $15 \%$ cases in each group.

\section{Discussion}

Rotator cuff pathology is a commonly encountered problem in the clinical setting. It is estimated that $30 \%-70 \%$ of all shoulder problems are cuff related. ${ }^{2,3}$ Cuff tears are debilitating, and therefore, accurate assessment and preoperative planning are of paramount importance in regaining maximum function and minimizing disability.

Historically, arthrography has been used in the past and is an excellent modality for detecting full-thickness tears. ${ }^{4}$ This is an invasive investigation with the possibility of complications. The advent of magnetic resonance imaging (MRI) scanning has superseded the need for arthrography to assess rotator cuff tears. The literature reports this to be a good modality with sensitivities $>90 \%$ and specificities $>80 \%{ }^{3,5,6}$ MRI scanning is, however, not performed immediately and takes longer time. Within the National Health Service sector in the UK, it can take many weeks. There are also obvious contraindications to perform an MRI scan. USS provides a quicker, cheaper, and more convenient alternative.

Published literature has reported a variation in the sensitivity and specificity of ultrasound scans in the diagnosis of both full-thickness and partial-thickness tears. Early results by Hedtmann and Fett showed a diagnostic accuracy

Table 2 Sensitivity and specificity of partial-thickness tears

\begin{tabular}{lll}
\hline Partial-thickness tears & Sensitivity & Specificity \\
\hline Overall results & $27 \%(\mathrm{Cl} \mathrm{I5 \% -43 \% )}$ & $86 \%(\mathrm{Cl} 79 \%-91 \%)$ \\
Radiographer & $17 \%(\mathrm{Cl} \mathrm{6 \% -35 \% )}$ & $94 \%(\mathrm{Cl} 88 \%-98 \%)$ \\
Radiologist & $50 \%(\mathrm{Cl} 23 \%-77 \%)$ & $70 \%(\mathrm{Cl} 55 \%-82 \%)$ \\
\hline
\end{tabular}

Abbreviation: $\mathrm{Cl}$, confidence interval.
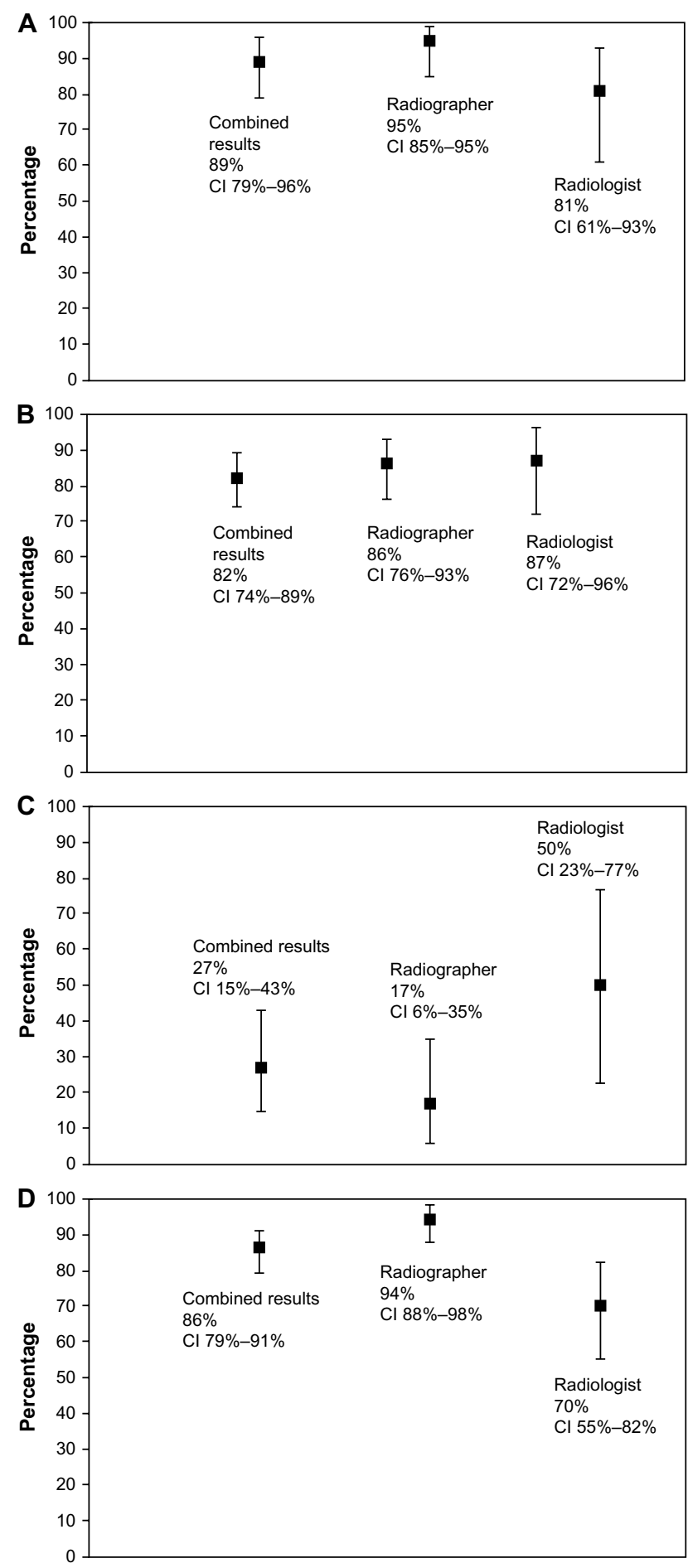

Figure I Sensitivities and specificities full and partial thickness tears.

Notes: (A) Sensitivity full-thickness tear. (B) Specificity full-thickness tear. (C) Sensitivity partial-thickness tear. (D) Specificity partial-thickness tear.

Abbreviation: $\mathrm{Cl}$, confidence interval.

of over $90 \%$ for full-thickness tears. ${ }^{7}$ Wallny et al reported an overall sensitivity of $91.3 \%$ and specificity of $82.3 \%$ for all cuff tears using conventional ultrasound but improved sensitivity to $>90 \%$ when three-dimensional scanning was performed. ${ }^{8}$ 
Table 3 Accuracy in quantifying the size of a full-thickness tear

\begin{tabular}{ll}
\hline & Accuracy \\
\hline Overall results & $43 \%$ \\
Radiographer & $55 \%$ \\
Radiologist & $23 \%$ \\
\hline
\end{tabular}

The results for partial-thickness tears are reported to be $<50 \%$ sensitivity, largely due to poor technique and expertise. ${ }^{4,9}$ Cullen et al compared the diagnostic USS performed by a single operator to surgical findings performed by a single surgeon in 68 cases. For full-thickness tears, the sensitivity was $89 \%$ and specificity was $100 \%$, and for partial-thickness tears, it was $79 \%$ and $94 \%{ }^{10}$

Experience in performing and interpreting any scan is always important and is considered more so in ultrasound. Moosmayer and Smith compared the results of an inexperienced surgeon performing scans in a clinic setting to the surgical findings and found a sensitivity of $77 \%$ and specificity of $98 \%$ in the diagnosis of full-thickness tears. Only one of the seven partial tears was identified at surgery. ${ }^{11}$ Jeyam et al compared a surgeon to a radiologist and found the sensitivity to detect full-thickness tears to be $92 \%$ and $94 \%$, respectively. For partial tears, the radiologist had a better sensitivity $(100 \%$ versus $85.7 \%)$, but the surgeon had a better specificity of $94 \%$ compared with $85 \%$ for the radiologist. ${ }^{12}$

Our outcomes for sensitivities and specificities, for fullthickness tears, do concur with existing literature, which suggests figures $>80 \%$. However for partial-thickness tears, the literature does report lower sensitivities and specificities. ${ }^{4,5}$ There are, however, exceptions. Vlychou et al ${ }^{6}$ reported sensitivities and specificities of $95.6 \%$ and $70 \%$, and Jeyam et $\mathrm{al},{ }^{12}$ as already stated earlier, reported sensitivities and specificities $>85 \%$. Ziegler reported figures $>92 \% .{ }^{13}$ Our sensitivities for partial-thickness tears were between $17 \%$ and $50 \%$. The specificities were between $70 \%$ and $94 \%$, suggesting

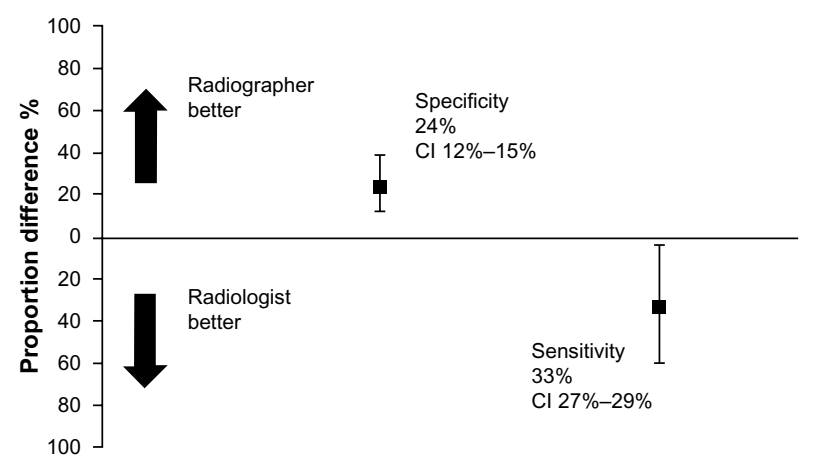

Figure 2 Percentage of proportion difference between the radiographer and radiologist for sensitivity and specificity in detecting partial-thickness tears. Abbreviation: $\mathrm{Cl}$, confidence interval. that for partial-thickness tears, ultrasound is less sensitive but more specific. This is in keeping with Martín-Hervás et $\mathrm{al}^{5}$ (sensitivity $-12.5 \%$ and specificity $-67.9 \%$ ) but not with others. $^{4}$

Our accuracy measured not only the absolute detection of a cuff tear but also the correct allocation into size category of $<1 \mathrm{~cm}, 1-3 \mathrm{~cm}, 3-5 \mathrm{~cm}$, and $>5 \mathrm{~cm}$. The overall accuracy rate was $43 \%$. There is no comparable literature to verify this against. Reported accuracy rates look at overall accuracy to detect tears but not quantify them. It should be noted that Kluger et al compared ultrasound and MRI to surgical findings, looking specifically at size and found no significant difference in ultrasound compared to MRI. Ultrasound has a tendency to underestimate tears $\geq 35 \mathrm{~mm}$ as well as a limitation to assess tears $>30 \mathrm{~mm} .{ }^{14}$ This may indicate the reason for the low figures. In our series, of the 70 full-thickness tears, 45 were $>3 \mathrm{~cm}$ at surgery.

Our study also looked at the impact of intraoperative change in plan. Previous studies have not mentioned the impact of this. There is clearly an implication with theater utilization and logistics. Although changes in the plan should be expected as no method of investigation is $100 \%$ reliable, it would be ideal to keep this to a minimum. We found that $15 \%$ of cases across the groups required an intraoperative change in the plan. We are unaware of comparable figures in other units.

There are obvious and clear limitations. Progression in the pathology of the rotator cuff is inevitable with time. Although the upper end of the waiting period was 12 weeks from the time of the scan to surgery, we do not know the extent of change in the condition of the rotator cuff during this period. The scan may have been accurate when it was undertaken. The impact of obesity and its limitations on the scans have not been evaluated. Arthroscopic evaluation is subject to observer bias and cannot assess intrasubstance tears that may have been detected on scans and reported as partial tears.

No previous study has compared a medically trained doctor with a radiographer. Our results do show that the radiographers have a better specificity for partial tears, but this is marginally correct with respect to the confidence intervals. The accuracy rates are much better for the radiographer with a proportional difference of $31 \%$ but with confidence intervals ranging from $8 \%$ to $51 \%$. However, with larger numbers, this may show a different result.

We can conclude that it is reasonable for an appropriately trained radiographer to perform an USS of the shoulder to aid in the diagnosis of cuff pathology. 


\section{Disclosure}

The authors report no conflicts of interest in this work.

\section{References}

1. Seltzer SE, Finberg HJ, Weissman BM, Kido DK, Collier BD. Arthrosonography: gray-scale ultrasound evaluation of the shoulder. Radiology. 1979;132:467-468.

2. Linsell L, Dawson J, Zondervan K, et al. Prevalence and incidence of adults consulting for shoulder conditions in UK primary care; patterns of diagnosis and referral. Rheumatology (Oxford). 2006;45(2):215-221.

3. Mitchell C, Adebajo A, Hay E, Carr A. Shoulder pain: diagnosis and management in primary care. BMJ. 2005;331:1124-1128.

4. Matsen FA, Arntz CT, Lippitt SB. Rotator cuff. In: Rockwood CA, Matsen FA, editors. The Shoulder. 2nd ed. Philadelphia: Saunders; 1998:789-791.

5. Martín-Hervás C, Romero J, Navas-Acién A, Reboiras JJ, Munuera L. Ultrasonographic and magnetic resonance images of rotator cuff lesions compared with arthroscopy or open surgery findings. J Shoulder Elbow Surg. 2001;10(5):410-415.

6. Vlychou M, Dailiana Z, Fotiadou A, Papanagiotou M, Fezoulidis IV, Malizos K. Symptomatic partial rotator cuff tears: diagnostic performance of ultrasound and magnetic resonance imaging with surgical correlation. Acta Radiol. 2009;50(1):101-105.

7. Hedtmann A, Fett H. Ultrasonography of the shoulder in subacromial syndromes with disorders and injuries of the rotator cuff. Orthopade. 1995;24(6):498-508.
8. Wallny TA, Schild RL, Schulze Bertelsbeck D, Hansmann ME, Kraft CN Three-dimensional ultrasonography in the diagnosis of rotator cuff lesions. Ultrasound Med Biol. 2001;27(6):745-749.

9. Teefey SA, Hasan SA, Middleton WD, Patel M, Wright RW, Yamaguchi K. Ultrasonography of the rotator cuff. A comparison of ultrasonographic and arthroscopic findings in one hundred consecutive cases. $J$ Bone Joint Surg Am. 2000;82(4):498-504.

10. Cullen DM, Breidahl WH, Janes GC. Diagnostic accuracy of shoulder ultrasound performed by a single operator. Australas Radiol. 2007; 51(3):226-229.

11. Moosmayer S, Smith HJ. Diagnostic ultrasound of the shoulder - a method for experts only? Results from an orthopedic surgeon with relative inexpensive compared to operative findings. Acta Orthop. 2005;76(4):503-508.

12. Jeyam M, Funk L, Harris J. Are shoulder surgeons any good at diagnosing rotator cuff tears using ultrasound?: a comparative analysis of surgeon vs radiologist. Int J Shoulder Surg. 2008;2(1):4-6.

13. Ziegler DW. The use of in-office, orthopaedist-performed ultrasound of the shoulder to evaluate and manage rotator cuff disorders. $J$ Shoulder Elbow Surg. 2004;13(3):291-297.

14. Kluger R, Mayrhofer R, Kröner A, et al. Sonographic versus magnetic resonance arthrographic evaluation of full-thickness rotator cuff tears in millimeters. J Shoulder Elbow Surg. 2003;12(2):110-116.
Orthopedic Research and Reviews

\section{Publish your work in this journal}

Orthopedic Research and Reviews is an international, peer-reviewed, open access journal focusing on the patho-physiology of the musculoskeletal system, trauma, surgery and other corrective interventions to restore mobility and function. Advances in new technologies, materials, techniques and pharmacological agents are particularly welcome. The journal welcomes

\section{Dovepress}

original research, clinical studies, reviews \& evaluations, expert opinion and commentary, case reports and extended reports. The manuscript management system is completely online and includes a very quick and fair peer-review system, which is all easy to use. Visit http://www.dovepress. com/testimonials.php to read real quotes from published authors. 